\title{
Periarteritis nodosa and thrombotic thrombocytopenic purpura with serous retinal detachment in siblings
}

\author{
F. H. STEFANI, F. BRANDT, AND K. PIELSTICKER \\ From the University Eye Hospital, Institute of Pathology, University of Munich
}

SUMMARY Periarteritis nodosa and thrombotic thrombocytopenic purpura in siblings is reported. In both patients a localised serous retinal detachment and lesions of the retinal pigment epithelium had developed owing to choroidal vascular obstruction. These cases support the suggested possible relationship between the two conditions.

Kussmaul and Maier (1866) coined the term periarteritis nodosa (PN). The disease is generally characterised by fever, leucocytosis, eosinophilia, abdominal crises, multiple capillary haemorrhages, thromboses, infarctions, signs of myositis, small firm subcutaneous nodules, renal and cardiac insufficiency, and cerebral symptoms. The term polyarteritisnodosa was introduced by Ferrari (1903). Intraocular involvement may occur and is generally due to retinal and choroidal disease (Sampson, 1945; Boeck, 1956).

In 1924 Moschcowitz first described a new vascular disease on a morphological basis and the next year published her case (Moschcowitz, 1925) under the descriptive title 'An acute febrile pleiochromic anaemia with hyaline thrombosis of the terminal arterioles and capillaries'. Subsequently Baehr et al. (1936) defined the clinical and morphological features of a disease which is now generally known as thrombotic thrombocytopenic purpura (TTP). The symptoms at first are non-specific. They then develop into an acute fever, with short attacks of cerebral symptoms, haemorrhages, haemolytic anaemia, varying degrees of thrombocytopenia, and disseminated small thrombi. As in PN, the eyes may be involved. Percival (1970) gave the first ophthalmic pathological report on the disease, and Cogan $(1975,1976)$ followed.

The aetiology of both diseases is unknown, but some relationship between the two has been suspected. This paper gives further support for a

Address for reprints: Dr F. H. Stefani, Augenklinik der Universität, Mathildenstrasse 8, D-8000 München 2, West Germany possible close relationship between both conditions. Their occurrence in the daughter and the son of healthy parents is reported.

\section{Case 1}

In 1974 a 23-year-old housewife was referred to the University Eye Hospital for routine examination because of arterial hypertension of renal origin. The hypertension was first observed 6 years previously after a history of pyelonephritis at the age of 14 years. Albuminuria of 1 to $2 \mathrm{~g}$ /day and bacteriuria together with a high blood pressure had led to an initial diagnosis of a chronic pyleonephritis without pyelographic and angiographic changes. Three months before referral she had suffered from pre-eclamptic toxaemia.

Ophthalmic examination showed normal visual acuity and unimpaired ocular motility. There was a slightly different intraocular tension in the 2 eyes ( $\mathrm{R}, 8 \mathrm{mmHg}$ applanation; $\mathrm{L}, 10 \mathrm{mmHg}$ applanation). The pupillary reactions were normal. Slit-lamp examination showed an increased number of cells in the aqueous but without corneal precipitates, changes of the stromal structure of the iris, or cells within the vitreous. Ophthalmoscopy showed a stage II hypertensive retinopathy, optic discs of normal appearance but no foveal reflex in either eye. There was an irregularity of the retinal pigment epithelium at the right posterior pole and a localised retinal detachment in the upper temporal quadrant, with some adjacent pigmentary irregularities (Figs. 1 and 2). Fluorescein angiography showed some leakage of the dye over the whole area of both optic discs and late-stage choroidal leakage under the 


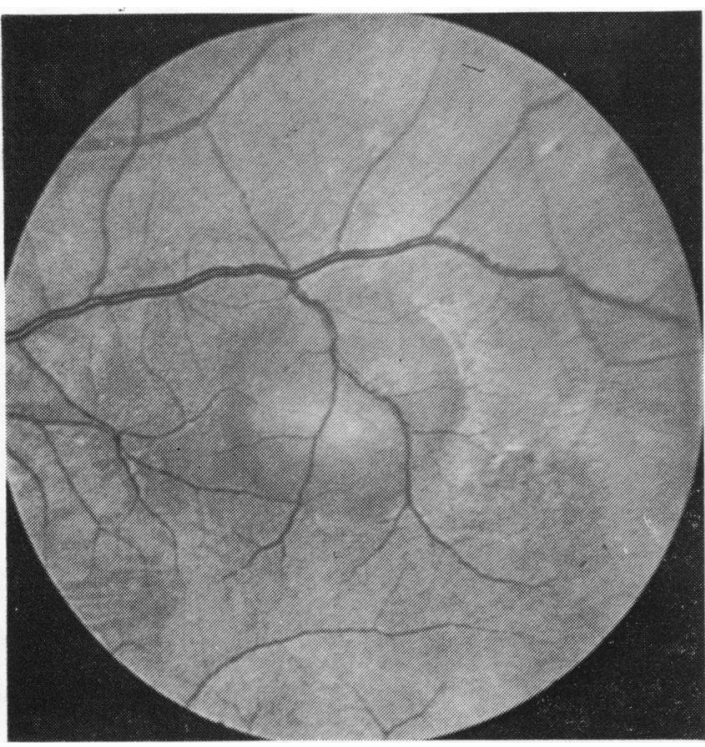

Fig. 1 Case 1: Localised retinal detachment with adjacent scar formation

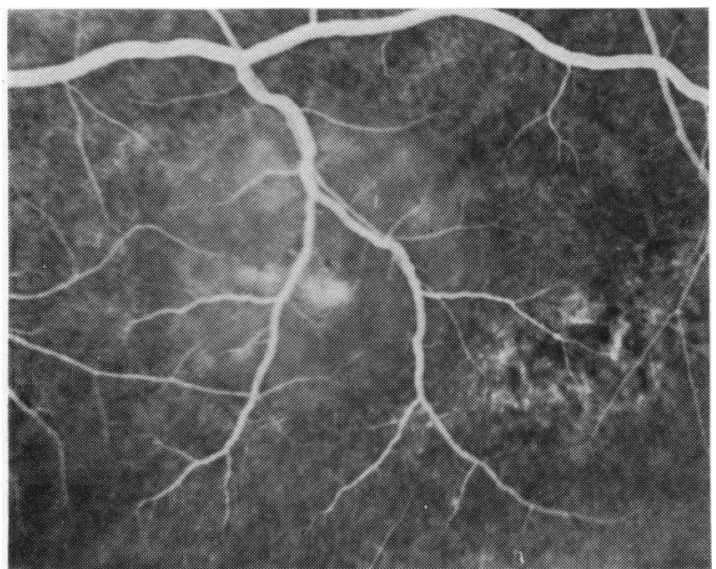

Fig. 2 Case 1: Fluorescein angiography showing some defects of the retinal pigment epithelium barrier and the adjacent scar

detached retinal area of the left eye (Fig. 2). As a result of treatment the blood pressure at this time was lowered to $150 / 120 \mathrm{mmHg}$.

One month later she developed numerous small, firm, painful inflammatory nodules in her legs. A skin biopsy (Fig. 3) led to the diagnosis of periarteritis nodosa. Serological tests were positive for rheumatoid arthritis and negative for lupus erythematosus and syphilis. She had no fever, anaemia, or skin haemorrhages. Platelets were not counted.
Under general corticosteroid therapy of initially $80 \mathrm{mg} /$ day the retina became re-attached and the skin lesions disappeared. But during an acute hypertensive crisis with a blood pressure of 280/ $180 \mathrm{mmHg}$ she developed a right hemiplegia and incomplete motoric aphasia. Antihypertensive treatment lowered the blood pressure and led to recovery.

In 1976 she had a period of melaena and was therefore admitted to another hospital. Platelets counted at this time were between 100000 and $160000 / \mu l\left(100\right.$ to $\left.160 \times 10^{9} / 1\right)$.

\section{DIAGNOSIS}

The picture was that of periarteritis nodosa as described by Kussmaul and Maier.

\section{Case 2}

In 1976 a 16-year-old boy was admitted to our hospital because of bilateral blurred vision of sudden onset 6 days previously. He gave a sketchy medical history of anaemia, with intervals of hearing difficulties, and allergic reactions against penicillins and fruits. He was not aware of the previous hospital treatment of his elder married sister (Case 1).

On admission visual acuity was $5 / 5$ in both eyes. The morphology of the anterior segment was normal and the media were clear. The posterior pole in both eyes showed multiple small yellow dots (Figs. 4 and 5), and the first diagnosis was of some form of a flecked retina syndrome. The erythrocyte sedimentation rate was 5 and $15 \mathrm{~mm}$ in the first and second hours and haemoglobin $9 \mathrm{~g} / \mathrm{dl}$. On the following day he had developed a bilateral serous retinal detachment round the optic disc (Figs. 4-6).

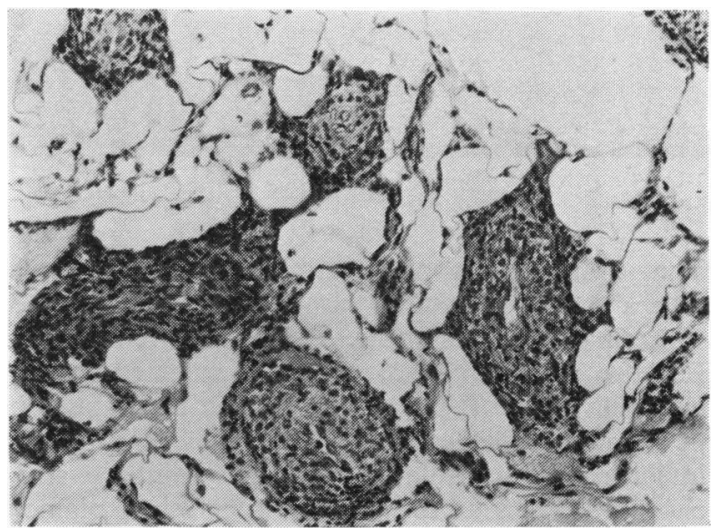

Fig. 3 Case 1: Skin biopsy from an inflammatory nodule of the leg, showing medium-sized arteries with inflammatory infiltrations and stenosis. (H and $\mathrm{E})$ 


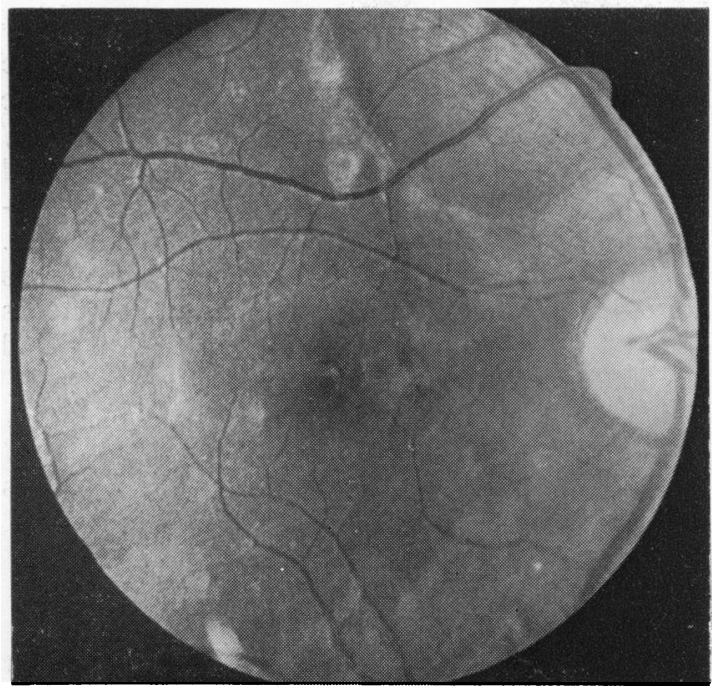

Fig. 4 Case 2: Fundus appearance of the right eye with small yellow dots and localised retinal detachment

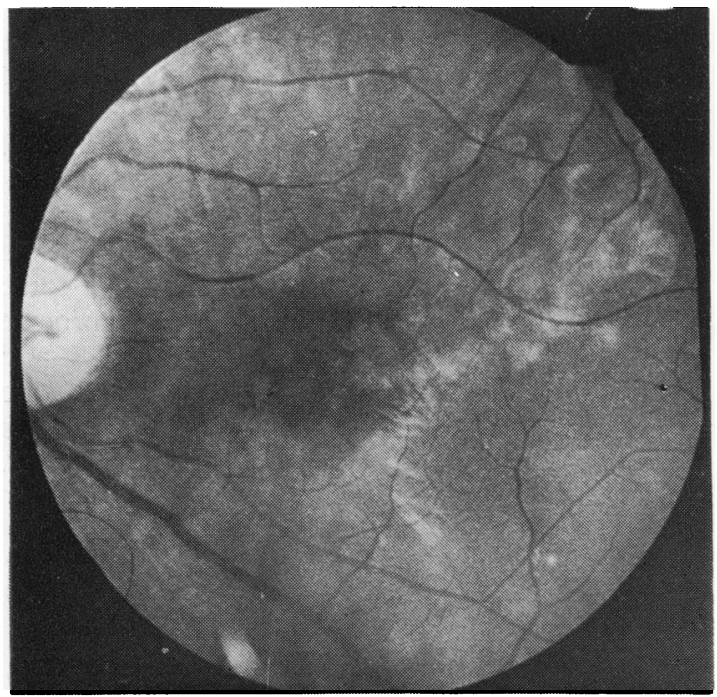

Fig. 5 Case 2: Fundus appearance of the left eye

The ophthalmoscopic appearance was that of several localised, round, rather sharply demarcated subretinal exudates with a tendency to confluence. Because a systemic process was suspected therapy was delayed until further diagnostic tests could be performed. Fluorescein angiography showed an intact retinal vascular system but multiple small choroidal leaks at the level of the retinal pigment epithelium related to the ophthalmoscopically observed yellow dots at the posterior pole (Fig. 7).

Without any specific treatment the ocular com- plant ceased during the next 2 days of observation, after which time the patient suddenly complained of paraesthesias in both hands lasting about half an hour. Neurological examination revealed no abnormalities. Three hours later he developed abdominal pains and vomiting without any other clinical signs. Some hours later he suddenly became unconscious, and arterial hypertension, oliguria, and a thrombocytopenia of $21000 / \mu 1\left(21 \times 10^{9} / 1\right)$ was noticed. Despite all therapeutic efforts the patient died from cardiac arrest. After his death further details of his clinical history became available, these indicating that he had suffered from chronic non-sphaerolytic haemolytic anaemia with thrombocytopenia and hepatosplenomegaly. He had been treated in hospital three times before because of abdominal pain and purpura.

\section{GENERAL PATHOLOGY}

At necropsy multiple disseminated small scars and

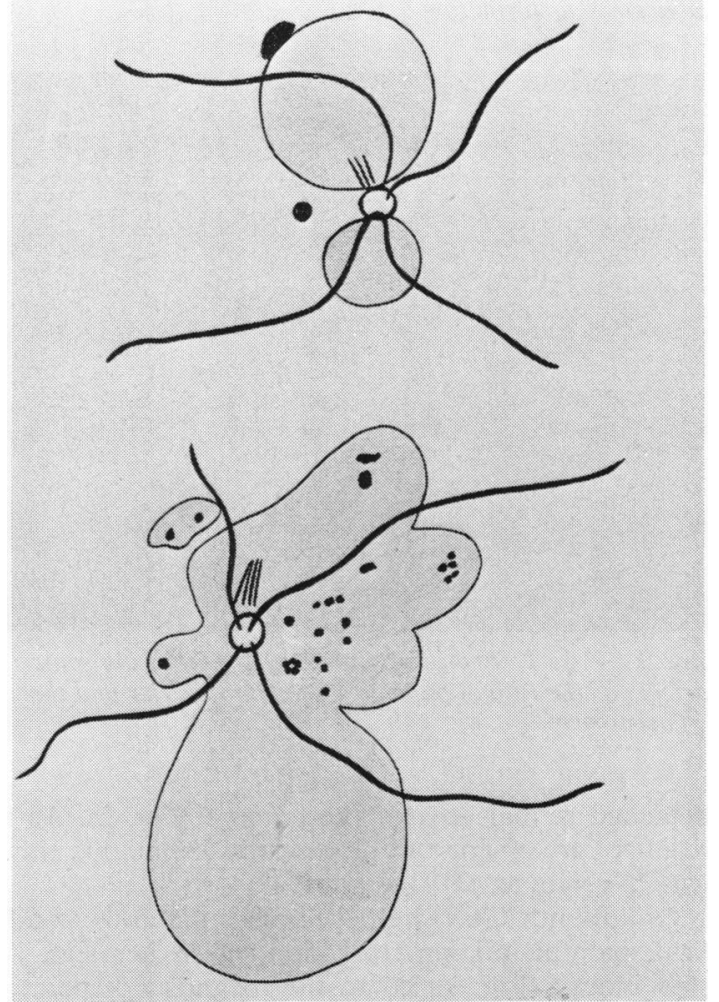

Fig. 6 Case 2: Schematic drawing of the localised retinal detachment (grey area) round the optic disc in both eyes, with some small yellow dots (black spots), and a small superficial retinal haemorrhage adjacent to the optic disc (black lines) 
Fig. 7 Case 2: Multiple defects of the retinal pigment epithelium barrier demonstrated in fluorescein angiography

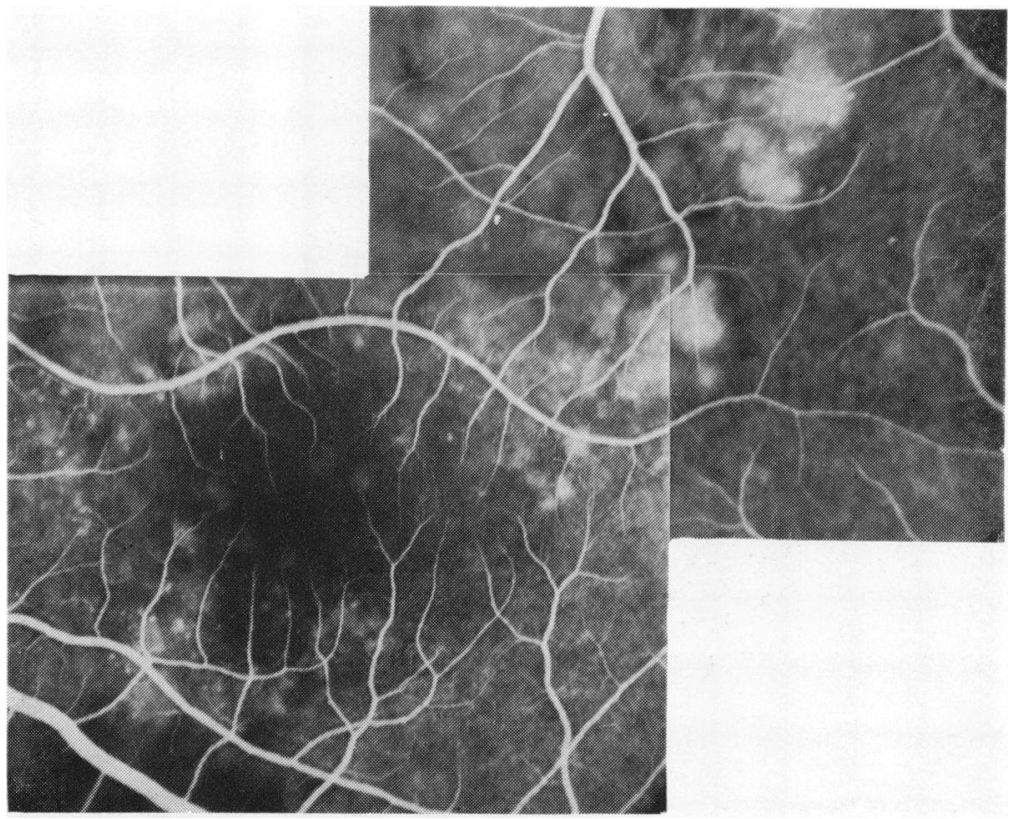

fresh necroses were observed within the brain, heart, kidneys, suprarenal glands, and spleen. There was a moderate hepatosplenomegaly and a slight enlargement of the lymph nodes. Histological examination revealed multiple hyaline thrombi within small arteries, arterioles, and capillaries as well as a thrombotic glomerulopathy of the kidney, some siderosis of the spleen and liver and extramedullary haematopoiesis, histiocytosis within the sinuses of the lymph nodes, and some phagocytosis of erythrocytes.

\section{OCULAR PATHOLOGY}

Macroscopical examination of both globes (measurements $24 \times 24 \times 23.5 \mathrm{~mm}$ ) showed localised serous retinal detachments round the optic disc and beneath the macula plus autolytic folding of the retina.

Microscopically there was an extensive PASpositive and Mallory's PTAH-positive fibrinous occlusion of the choriocapillaris at the posterior pole (Fig. 8) extending into larger vessels of the choroid (Figs. 9 and 10). The retinal pigment epithelium showed a higher degree of necrotic changes than could be explained by autolytic changes, and there was a subretinal exudate in places (Fig. 10). Some scattered round cells and erythrocytes were found within the posterior choroid without any evidence of an angiitis. A calcifying thrombus in the choroid (Fig. 11) was regarded as a sign of previous choroidal involvement.
DIAGNOSIS

The picture was that of thrombotic thrombocytopenic purpura as described by Moschcowitz.

\section{Discussion}

Serous retinal detachment due to choroidal blood vessel occlusions is a well-recognised finding in a variety of clinical conditions, such as malignant arterial hypertension (Wagener, 1929), especially when complicating renal diseases (Koyanagi, 1934; Buchanan and Ellis, 1964; Paris and Macoul, 1969), eclampsia (Fry, 1929; Gitter et al., 1968), disseminated intravascular coagulation (Cogan, 1976), PN (Sampson, 1945), and TTP (Percival, 1970; Cogan, 1976). It has been produced experimentally by embolisation into the choroidal circulation (Algvere, 1976). Since the anatomical studies of Wybar (1954), and Ring and Fujino (1967) the predilection of vascular lesions for the posterior pole of the eye has had a probable explanation in the anatomical and haemodynamic pecularities of this area.

The detachment is related to lesions of the retinal pigment epithelium, changes in which are observed ophthalmoscopically as isolated, round, light yellowish spots of $1 / 6$ to $1 / 3$ disc diameters ( 3 to 4 times the diameter of a retinal artery) with a pigmented centre (Elschnig spots). Such lesions are recognised clinically as a grave prognostic sign in occlusive vascular diseases of the choroid (Klien, 1968). Experimentally as soon as 1 hour after microsphere 


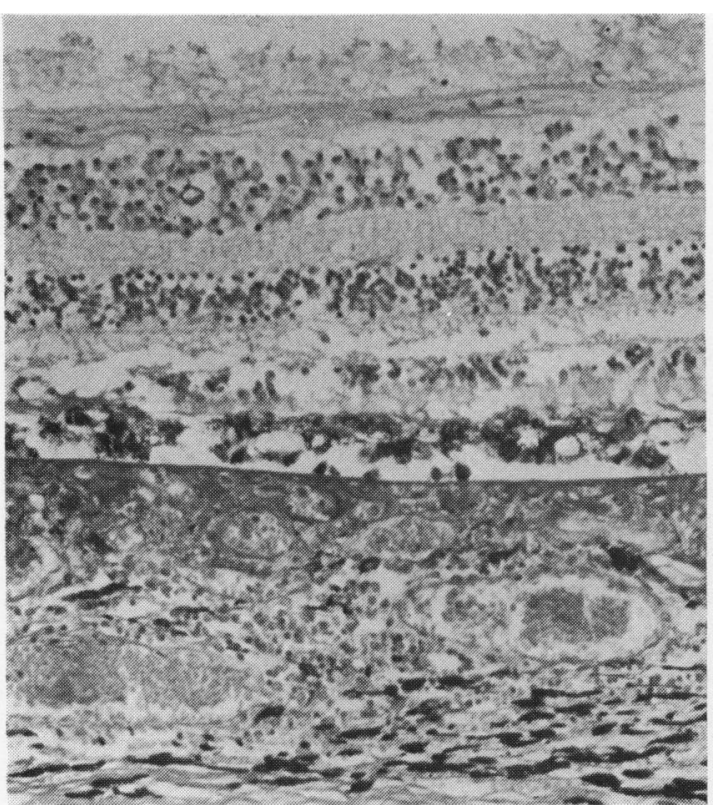

Fig. 8 Case 2: Massive fibrinous occlusion of the choriocapillary with lesions of the retinal pigment epithelium (Periodic acid Schiff)

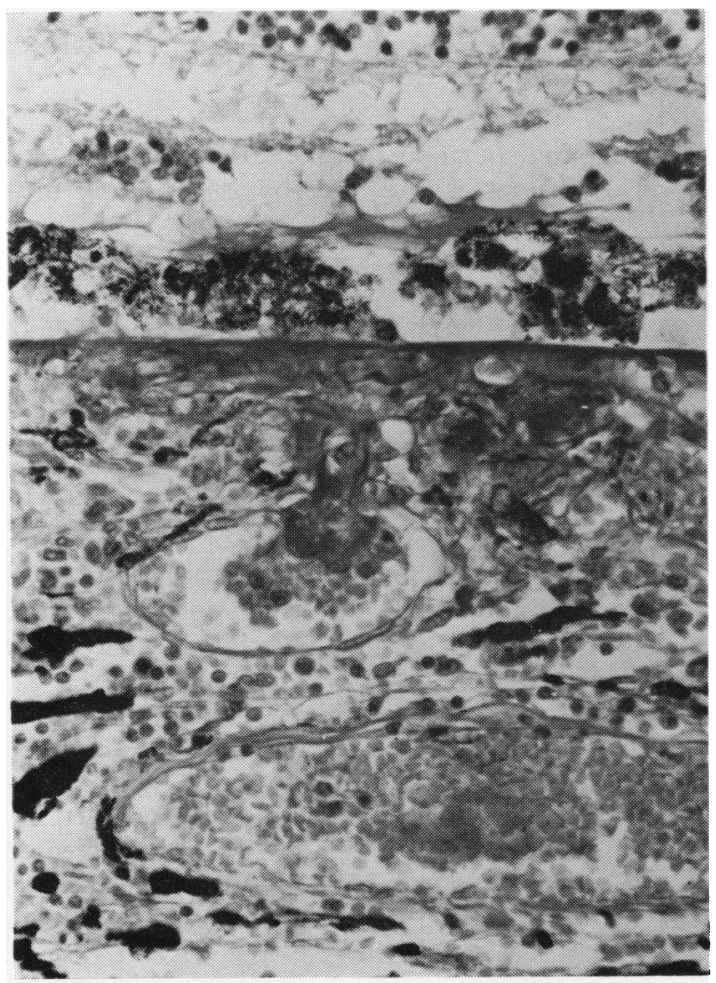

Fig. 9 Case 2: Higher magnification of Fig. 8

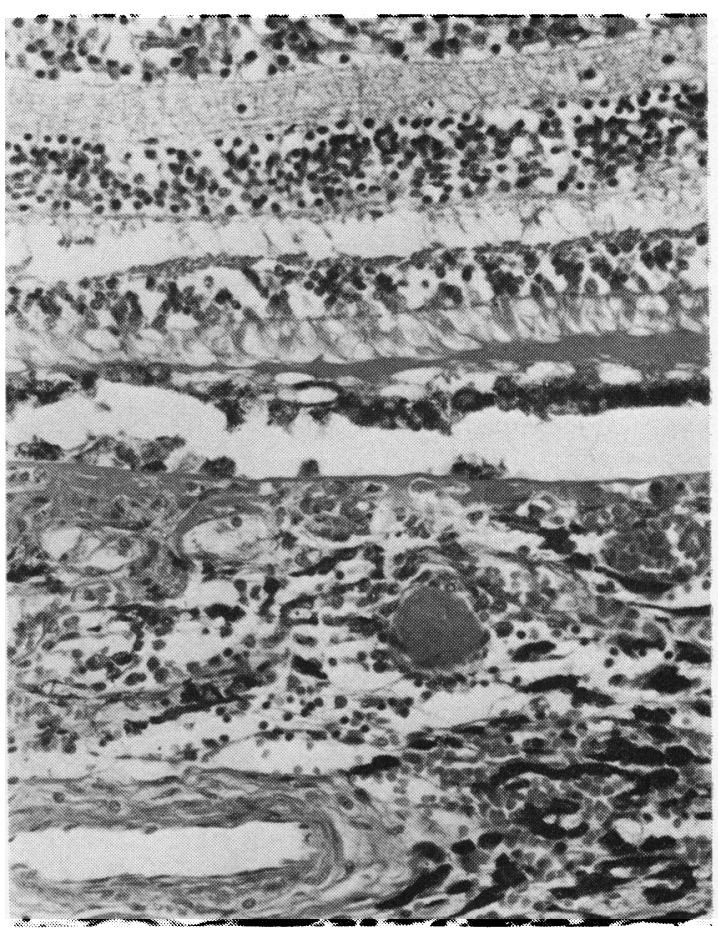

Fig. 10 Case 2: Extension of the fibrinous occlusion into medium-sized choroidal blood vessels. Subretinal exudate (PAS)

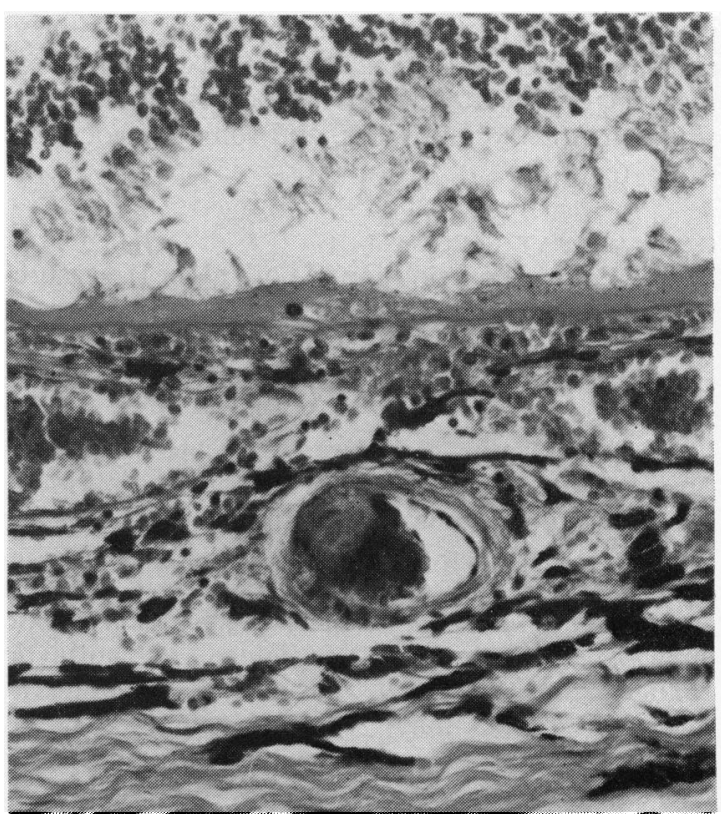

Fig. 11 Case 2: Calcified thrombus in a choroidal blood vessel (PAS) 
embolisation of the choriocapillaris (Sterne and Ernest, 1974) lesions of the retinal pigment epithelium were visible.

In our 2 cases there was clinical and microscopic evidence of PN and TTP. In PN the inflammatory lesion of the blood vessels leads to occlusions. In TTP the underlying mechanism is as yet unknown; it may be intravascular coagulation, or it may be a primary vascular disease. In neither disease can significant alterations in the clotting mechanisms be demonstrated. The most popular hypothesis for the aetiology of both diseases is an autoimmune mechanism. Benitez et al. (1964) reported the simultaneous occurrence of PN and TTP in a patient. Although familial occurrence of PN has been observed, there is to our knowledge no publication on PN and TTP in siblings which would support the suggested association of both conditions. Both may show a very similar ophthalmoscopic resemblance to central serous retinopathy (Wolkowicz, 1956). Unilateral eccentric circumscribed serous retinal detachment or bilateral circumscribed serous retinopathy may be important signs in the recognition of a disease likely to be fatal if untreated.

We gratefully acknowledge permission granted by the University Hospital of Dermatology, Munich (Head: Professor Dr O. Braun-Falco) to use slide No. 74/3172 for the presentation of Fig. 3.

\section{References}

Algvere, P. (1976). Retinal detachment and pathology following experimental embolization of choroidal and retinal circulation. Albrecht von Graefes Archiv für klinische und experimentelle Ophthalmologie, 201, 123-134.

Baehr, G., Klemperer, P., and Schiffrin, A. (1936). Acute febrile anemia and thrombocytopenic purpura with diffuse platelet thromboses of capillaries and arterioles. Transactions of the Association of American Physicians, 51, 43-58.

Benitez, L., Mathews, M., and Mallory, G. K. (1964). Platelet thrombosis with polyarteritis nodosa. Archives of Pathology, 77, 116-125.

Boeck, J. (1956). Ocular changes in periarteritis nodosa. American Journal of Ophthalmology, 42, 567-576.

Buchanan, W. S., and Ellis, P. P. (1964). Retinal separation in chronic glomerulonephritis. Archives of Ophthalmology, 71, 182-186.

Cogan, D. G. (1975). Ocular involvement in disseminated intravascular coagulopathy. Archives of Ophthalmology, 93, 1-8.

Cogan, D. G. (1976). Fibrin clots in the choriocapillaris and serous detachment of the retina. Ophthalmologica, 172, 298-307.

Ferrari, E. (1903). Über Polyarteritis acuta nodosa (sogenannte Periarteritis nodosa) und ihre Beziehungen zur Polymyositis und Polyneuritis acuta. Beiträge zur Pathologischen Anatomie und zur allgemeinen Pathologie, 24, 350-386.

Fry, W. E. (1929). Extensive bilateral retinal detachment in eclampsia with complete reattachment: Report of two cases. Archives of Ophthalmology, 1, 609-614.

Gitter, K. A., Houser, B. P., Sarin, L. K., and Justice, J., Jr. (1968). Toxemia of pregnancy. An angiographic interpretation of fundus changes. Archives of. Ophthalmology, 80, 449-454.

Klien, B. A. (1968). Ischemic infarcts of the choroid (Elschnig spots). American Journal of Ophthalmology, 66, 1069-1074.

Koyanagi, Y. (1934). Über die Herkunft und Bedeutung des subretinalen Ergusses bei Retinitis nephritica. Albrecht von Graefes Archiv für Ophthalmologie, vereinigt mit Archiv für Augenheilkunde, 132, 353-369.

Kussmaul, A., and Maier, R. (1866). Über eine bisher nicht beschriebene Arterienerkrankung (Periarteritis nodosa), die mit Morbus Brightii und mit rapid fortschreitender allgemeiner Muskellähmung einhergeht. Deutsches Archiv für Klinische Medizin, 1, 484-518.

Moschcowitz, E. (1925). An acute febrile pleiochromic anemia with hyaline thrombosis of the terminal arterioles and capillaries. Archives of Internal Medicine, 36, 89-93.

Paris, G. C., and Macoul, K. L. (1969). Reversible bullous retinal detachment in chronic renal disease. American Journal of Ophthalmology, 67, 249-251.

Percival, S. P. B. (1970). The eye and Moschcowitz's disease (thrombotic thrombocytopenia). A review of 182 cases. Transactions of the Ophthalmological Societies of the United Kingdom, 90, 375-382.

Percival, S. P. B. (1970). Ocular findings in thrombotic thrombocytopenic purpura (Moschcowitz's disease). British Journal of Ophthalmology, 54, 73-78.

Ring, H. G., and Fujino, T. (1967). Observations on the anatomy and pathology of the choroidal vasculature. Archives of Ophthalmology, 78, 431-444.

Sampson, R. (1945). Periarteritis nodosa affecting the eye. British Journal of Ophthalmology, 29, 282-288.

Sterne, W. H., and Ernest, J. T. (1974). Microsphere occlusion of the choriocapillaris in rhesus monkeys. American Journal of Ophthalmology, 78, 438-448.

Wagener, H. P. (1929). The retinitis of malignant hypertension. Transactions of the American Ophthalmological Society, 25, 349-380.

Wolkowicz, M. I. (1956). Central serous retinopathy. Clinical and experimental studies. American Journal of Ophthalmology, 42, 531-545.

Wybar, K. C. (1954). Vascular anatomy of the choroid in relation to selective localization of ocular disease. British. Journal of Ophthalmology, 38, 513-527. 